\section{DNMT3A mutation is associated with increased age and adverse outcome in adult T-cell acute lymphoblastic leukemia}

Jonathan Bond,,${ }^{1,2,3}$ Aurore Touzart, ${ }^{1}$ Stéphane Leprêtre, ${ }^{4}$ Carlos Graux, ${ }^{5}$ Mario Bargetzi, ${ }^{6,7}$ Ludovic Lhermitte, ${ }^{1}$ Guillaume Hypolite, ${ }^{1}$ Thibaut Leguay, ${ }^{8}$ Yosr Hicheri, ${ }^{9}$ Gaëlle Guillerm, ${ }^{10}$ Karin Bilger, ${ }^{11}$ Véronique Lhéritier, ${ }^{12}$ Mathilde Hunault, ${ }^{13}$ Françoise Huguet, ${ }^{14}$ Yves Chalandon, ${ }^{6,15}$ Norbert Ifrah, ${ }^{13}$ Elizabeth Macintyre, ${ }^{1}$ Hervé Dombret, ${ }^{16}$ Vahid Asnafi ${ }^{1}$ and Nicolas Boissel ${ }^{16}$

\begin{abstract}
${ }^{1}$ Université Paris Descartes Sorbonne Cité, Institut Necker-Enfants Malades (INEM), Institut National de Recherche Médicale (INSERM) U1151, and Laboratory of OncoHematology, Assistance Publique-Hôpitaux de Paris (AP-HP), Hôpital Necker EnfantsMalades, Paris, France; ${ }^{2}$ Systems Biology Ireland, School of Medicine, University College Dublin, Ireland; ${ }^{3}$ National Children's Research Centre, Children's Health Ireland at Crumlin, Dublin, Ireland; ' ${ }^{4}$ NSERM U1245 and Department of Hematology, Centre Henri Becquerel and Normandie Université UNIROUEN, Rouen, France; ${ }^{5}$ Department of Hematology, Université Catholique de Louvain (UCL), Centre Hospitalier Universitaire (CHU) Namur - Godinne site, Yvoir, Belgium; ${ }^{6}$ University Medical Department, Division of Oncology, Hematology and Transfusion Medicine, Kantonsspital Aarau, Aarau, Switzerland; ' Swiss Group for Clinical Cancer Research (SAKK), Bern, Switerland; ${ }^{8}$ Department of Hematology, CHU de Bordeaux, France; ${ }^{\circ}$ Hematology Service, Hôpital St Eloi, Montpellier, France; ${ }^{10}$ Hematology Service, $\mathrm{CHU}$ de Brest, Brest, France; ${ }^{11}$ Hematology Service, CHU Hautepierre, Strasbourg, France; ${ }^{12}$ Group for Research on Adult Acute Lymphoblastic Leukemia, Coordination Office, Centre Hospitalier Lyon Sud, Lyon, France; ${ }^{13}$ PRES LUNAM, CHU Angers Service des Maladies du Sang and CRCINA INSERM, Angers, France; ${ }^{14}$ Department of Hematology, CHU de Toulouse, Institut Universitaire du Cancer de Toulouse Oncopole, Toulouse, France; ${ }^{15}$ Department of Oncology, Hematology Division, University Hospital, Geneva, Switzerland and ${ }^{16}$ Université Paris Diderot, Institut Universitaire d'Hématologie, EA-3518, AP-HP, University Hospital Saint-Louis, Paris, France
\end{abstract}

\section{ABSTRACT}

T The prognostic implications of DNMT3A genotype in T-cell acute lymphoblastic leukemia are incompletely understood. We performed comprehensive genetic and clinico-biological analyses of T-cell acute lymphoblastic leukemia patients with DNMT3A mutations treated during the GRAALL-2003 and -2005 studies. Eighteen of 198 cases $(9.1 \%)$ had DNMT3A alterations. Two patients also had DNMT3A mutations in nonleukemic cell DNA, providing the first potential evidence of age-related clonal hematopoiesis in T-cell acute lymphoblastic leukemia. DNMT3A mutation was associated with older age (median 43.9 years vs. 29.4 years, $P<0.001$ ), immature $\mathrm{T}$-cell receptor genotype (53.3\% vs. $24.4 \%, P=0.016$ ) and lower remission rates $(72.2 \%$ mutated vs. $94.4 \%$ non-mutated, $P=0.006$ ). DNMT3A alterations were significantly associated with worse clinical outcome, with higher cumulative incidence of relapse (HR 2.33, 95\% CI: 1.05-5.16, $P=0.037$ ) and markedly poorer event-free survival (HR 3.22, 95\% CI: 1.81-5.72, $P<0.001$ ) and overall survival (HR 2.91, 95\% CI: $1.56-5.43, P=0.001)$. Adjusting for age as a covariate, or restricting the analysis to patients over 40 years, who account for almost $90 \%$ of DNMT3Amutated cases, did not modify these observations. In multivariate analysis using the risk factors that were used to stratify treatment during the GRAALL studies, DNMT3A mutation was significantly associated with shorter event-free survival (HR 2.33, 95\% CI: 1.06 - 4.04, $P=0.02$ ). Altogether, these results identify DNMT3A genotype as a predictor of aggressive T-cell acute lymphoblastic leukemia biology. The GRAALL-2003 and -2005 studies were registered at http://www. ClinicalTrials.gov as \#NCT00222027 and \#NCT00327678, respectively.
Ferrata Storti Foundation

\section{Correspondence:}

NICOLAS BOISSEL

nicolas.boissel@aphp.fr

Received: May 24, 2018.

Accepted: January 10, 2019.

Pre-published: January 17, 2019.

doi:10.3324/haematol.2018.197848

Check the online version for the most updated information on this article, online supplements, and information on authorship \& disclosures: www.haematologica.org/content/104/8/1617

\section{(C)2019 Ferrata Storti Foundation}

Material published in Haematologica is covered by copyright. All rights are reserved to the Ferrata Storti Foundation. Use of published material is allowed under the following terms and conditions:

https://creativecommons.org/licenses/by-nc/4.0/legalcode. Copies of published material are allowed for personal or internal use. Sharing published material for non-commercial purposes is subject to the following conditions:

https://creativecommons. org//icenses/by-nc/4.0/legalcode, sect. 3. Reproducing and sharing published material for commercial purposes is not allowed without permission in writing from the publisher. 


\section{Introduction}

Mutations in the DNA methyltransferase 3 alpha gene (DNMT3A) have been reported in a range of hematologic malignancies, most frequently in myeloid neoplasia, including acute myeloid leukemia (AML) ${ }^{1.5}$ myelodysplastic syndromes, ${ }^{6}$ myeloproliferative neoplasms ${ }^{7}$ and myeloproliferative neoplasm/myelodysplastic overlap syndromes. ${ }^{8,9}$ DNMT3A alterations in lymphoid malignancies are less common, and reports to date are confined to T-lineage disease. ${ }^{10-16}$ In all cases, DNMT3A mutations increase in frequency with age, and are extremely rare in children and adolescents. ${ }^{17-19}$

Multiple studies have reported that DNMT3A alterations correlate with poor outcome in AML. . $2,2,202022^{2}$ In comparison, the prognostic influence of DNMT3A mutation in T-cell acute lymphoblastic leukemia (T-ALL) is poorly characterized. Patients with DNMT3A alterations were reported to have shorter survival in three moderately sized (55 to 93 patients) T-ALL cohorts., ${ }^{911,13}$ DNMT3A status did not however independently predict prognosis in the only series for which multivariate analyses were documented, as survival effects were linked to increased rates of DNMT3A mutation in poor-risk, phenotypically immature disease. ${ }^{11}$ While that study did document a correlation between DNMT3A alteration and survival within the immature T-ALL subgroup, this finding was not corroborated in an independent cohort of early thymic precursor (ETP) ALL cases. ${ }^{12}$

The issue of whether DNMT3A mutation truly alters the biology of T-ALL is therefore only partially addressed by the currently available evidence. In particular, it is unclear whether the associated poor survival simply reflects the prosaic fact that patients with DNMT3A alterations are older, ${ }^{11,12}$ and therefore do not tolerate intensive ALL treatment as well as their younger counterparts.

In order to address this question, we used next-generation sequencing (NGS) to evaluate the DNMT3A genotype of a large cohort of 198 adult T-ALL patients treated as part of the multinational GRAALL-2003 and -2005 studies. We found that DNMT3A mutation strongly correlated with disease relapse and shorter survival, and that these prognostic effects were independent of patients' age. Furthermore, we report the presence of DNMT3A mutations in nonleukemic cells in a subset of patients, providing the first evidence of age-related clonal hematopoiesis in T-ALL.

\section{Methods}

\section{Patients}

Details of the GRAALL-2003 and -2005 studies are provided in the Online Supplementary Methods. Informed consent was obtained from all patients before inclusion into the trials. Both studies were conducted in accordance with the Declaration of Helsinki and approved by local and multicenter research ethical committees. The complete study protocols are detailed in the Online Data Supplement. Both trials were registered at http:/www.ClinicalTrials.gov (NCT00222027, NCT00327678). The criteria for inclusion in the current project were a diagnosis of $\mathrm{T}$ ALL and the availability of diagnostic material for NGS analysis of DNMT3A genotype. Survival outcomes of the 198 patients (36 from GRAALL-2003 and 162 from GRAALL-2005) who fulfilled these criteria did not differ from those of the remaining 139 T-ALL patients of the study cohorts. As expected in retro- spective studies, initial white blood cell count (WBC) was higher in the study cohort. However, no differences in allogeneic stem cell transplant rate, disease-free survival, event-free survival, or overall survival were found. A full comparison of the clinical features of each group is shown in Online Supplementary Table S1.

\section{Next-generation sequencing}

Nextera XT (Illumina) DNA Libraries were prepared according to the manufacturer's instructions and sequenced using the Illumina MiSeq sequencing system. The custom NGS panel comprised genes coding for factors involved in molecular pathways known to be mutated in T-ALL, namely cytokine receptor and RAS signaling (NRAS, KRAS, JAK1, JAK3, STAT3, STAT5B, IL7R, BRAF, NF1, SH2B3, PTPN11), hematopoietic development (RUNX1, ETV6, GATA3, IKZF1, EP300), chemical modification of histones (SUZ12, EED, EZH2, KMT2A, KMT2D, SETD2) and DNA methylation (DNMT3A, IDH1, IDH2, TET2, TET3). This panel was originally inspired by the repertoire of genes found to be preferentially altered in pediatric ETP-ALL ${ }^{23}$ and we have reported a subset of the results described in the current paper in a previous clinico-biological and genetic analysis of adult ETPALL. ${ }^{24}$ Sequencing reads were analyzed using in-house software (Polyweb, Institut Imagine, Paris, France), and additional inhouse custom filtering criteria (comprising minimum read counts and variant allele frequencies, and reference to external reference databases) were applied to minimize false-positive rates. Primers used to confirm mutations by direct sequencing are listed in Online Supplementary Table S2.

\section{Outcome analyses}

Comparisons between groups were performed with the Fisher exact and Mann-Whitney tests for categorical and continuous variables respectively. Corticosteroid sensitivity was defined as clearance of peripheral blood circulating blasts $(<1 \mathrm{x}$ $10^{9} / \mathrm{L}$ ) following steroid prophase treatment. Complete remission was defined as clearance of bone marrow blasts $(<5 \%)$ following induction treatment. Overall survival was calculated from the date of inclusion in the trial to the last follow-up date, censoring patients alive at that date. Event-free survival was calculated from date of inclusion in the trial to the date of induction failure, relapse, or death, censoring patients alive in first complete remission without relapse at the last follow-up date Cumulative incidence of relapse was calculated in patients who attained complete remission, from the date of achieving the complete remission to the date of relapse, with death in first complete remission being considered as a competing event. Univariate and bivariate analyses assessing the impact of DNMT3A mutations and age were performed with a Cox model. Variables that were significantly associated with outcome in univariate analysis were considered as covariates in multivariate Cox models. The proportional-hazards assumption was checked before conducting multivariate analyses. Statistical analyses were performed with STATA software (STATA 12.0 Corporation, College Station, TX, USA). All $P$ values were twosided, with $P<0.05$ denoting statistical significance.

\section{Results}

Analysis of DNMT3A genotype in patients with T-cell
acute lymphoblastic leukemia in the GRAALL studies acute lymphoblastic leukemia in the GRAALL studies
We performed targeted NGS of a panel of genes, including DNMT3A, which have been described to be recurrently mutated in T-ALL. This panel included all exons of $D N M T 3 A$, thereby providing a comprehensive picture of 
the spectrum of alterations across this gene in T-ALL. Diagnostic DNA was available for 198 patients treated during the GRAALL-2003 and -2005 studies. A partial analysis of a subgroup of this cohort has been reported previously. ${ }^{24}$ We detected 21 DNMT3A mutations in 18 patients $(9.1 \%)$. Most alterations occurred in regions coding for defined protein functional domains, including six mutations at the R882 hotspot ${ }^{1}$ (Figure 1A). Further details of patient-specific alterations are shown in Online Supplementary Table S3. Of note, the vast majority of detected mutations are predicted to be significantly damaging to protein function.

In keeping with previous reports of DNMT3A-mutated
T-ALL which cited high rates of either compound heterozygosity or homozygosity, ${ }^{9,11}$ a significant proportion of cases (8/18) had either two separate alterations, or high variant allele frequencies that were suggestive of either homozygous mutation, concomitant deletion of the wildtype (WT) allele or copy-neutral loss of heterozygosity. Comparative genomic hybridization analyses were available for 85 of the cases in this study, including 6/18 patients with DNMT3A mutations. We detected only two deletions of the DNMT3A locus, which in each case were associated with concomitant DNMT3A mutation and elevated variant allele frequencies (cases 11 and 12 in Online Supplementary Table S3).

A

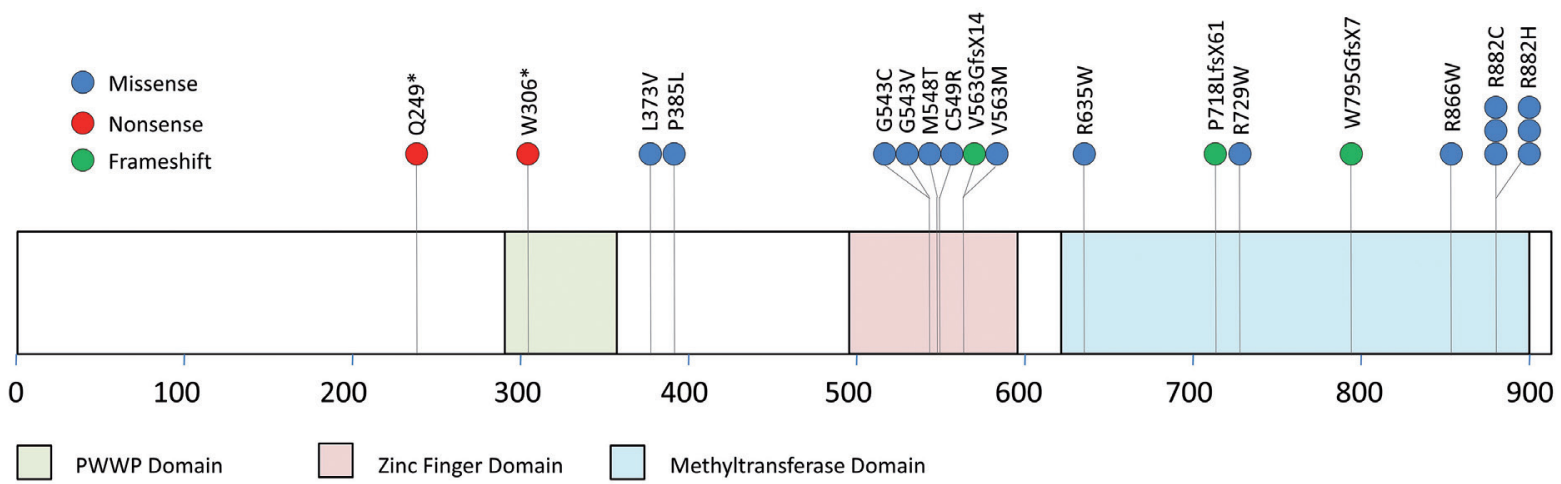

B

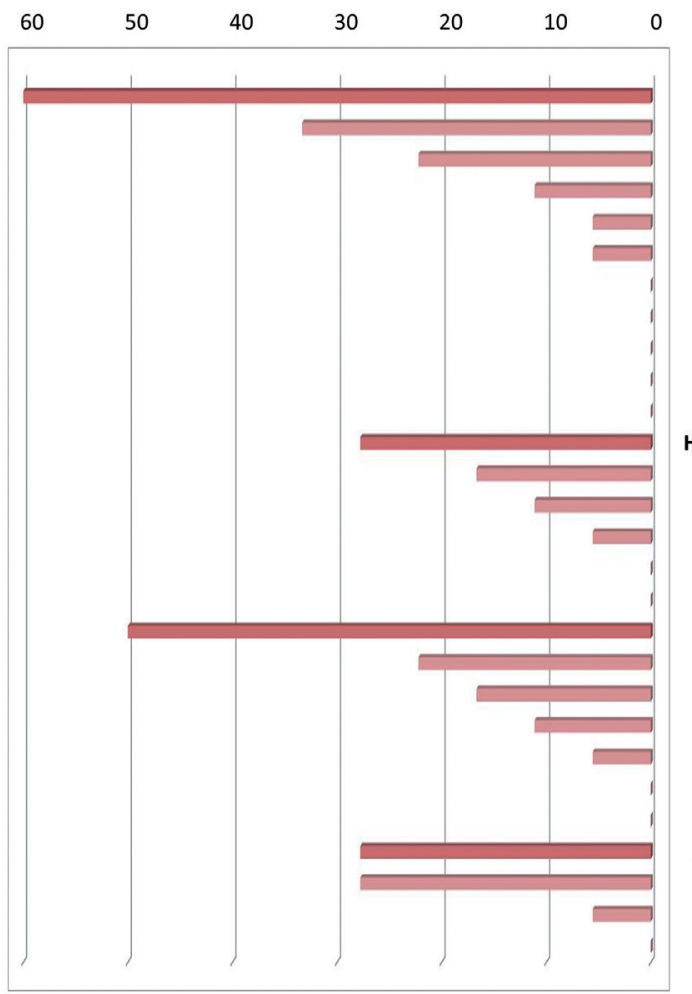

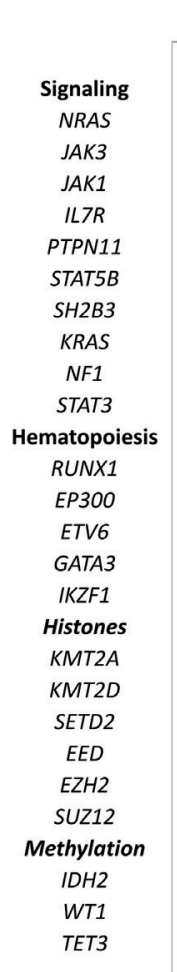

\% Frequency DNMT3A WT

$\begin{array}{lllllll}0 & 10 & 20 & 30 & 40 & 50 & 60\end{array}$

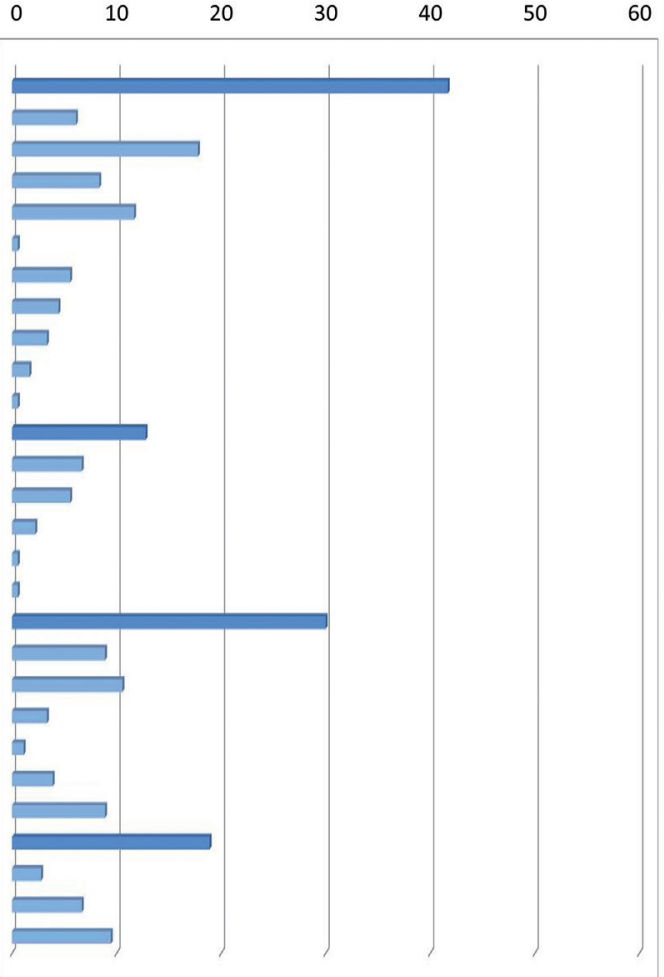

Figure 1. DNMT3A mutations in T-cell acute lymphoblastic leukemia. (A) Schematic representation of the 21 mutations detected in this study. Further patient-specific details are provided in Online Supplementary Table S3. (B) Comparison of the mutational genotypes of DNMT3A altered ( $\mathrm{n}=18$ ) and DNMT3A wild-type ( $\mathrm{n}=180$ ) T-cell acute lymphoblastic leukemia. Percentage frequencies in each group are depicted. Functional categories are listed in bold. 
The prevalence of other mutations detected by NGS is shown in Figure 1B. DNMT3A-altered cases had an increased frequency of alterations in other genes included in the NGS panel, compared with the rest of the cohort (88.9\% DNMT3A mutated vs. 64.4\% DNMT3A WT, $P=0.036)$. There were no statistically significant differences in the prevalence of mutations in any specific functional gene category, namely factors involved in cytokine receptor and RAS signaling (61.1\% DNMT3A mutated vs. $41.7 \%$ DNMT3A WT, $P=0.113)$, hematopoiesis $(38.5 \%$ DNMT3A mutated vs. $12.8 \%$ DNMT3A WT, $P=0.082)$ and chemical modification of histones $(50.0 \%$ DNMT3A mutated vs. $30.0 \%$ DNMT3A WT, $P=0.082)$. However, we did observe significant co-occurrence of DNMT3A alterations and IDH2 mutations (27.8\% DNMT3A mutated vs. $2.2 \%$ DNMT3A WT, $P<0.001)$. This association has been described previously in both $\mathrm{AML}^{25}$ and myelodysplastic syndromes. ${ }^{26}$

\section{Evidence of possible clonal hematopoiesis in DNMT3A- mutated T-cell acute lymphoblastic leukemia}

$D N M T 3 A$ is the most commonly altered gene in agerelated clonal hematopoiesis ${ }^{27-29}$ and DNMT3A mutations have been detected in non-malignant cells in $\mathrm{AML}^{30,31}$ and peripheral T-cell lymphoma. ${ }^{14}$ We therefore tested whether DNMT3A mutations were present in nonleukemic hematopoietic cells in T-ALL patients.

DNA from remission bone marrow was available for only three of the 18 patients with DNMT3A mutations. While two of these samples had a DNMT3A WT genotype (Online Supplementary Figure S1), one interesting case had evidence of DNMT3A alterations in non-leukemic bone marrow cells. At diagnosis, this patient (case 6 in Online Supplementary Table S3) had mutations in exons 14 and 15 of DNMT3A, a NOTCH1 PEST domain insertion, and an NRAS G12D substitution. Sequencing of remission DNA revealed mutation of DNMT3A exon 14 in nonleukemic cells, while NOTCH1, NRAS and DNMT3A exon 15 all presented wild-type genotypes (Figure 2A). We confirmed that the exon 14 mutation has never been reported as a polymorphic variant, while SIFT analysis (http://sift.jcvi.org) predicted this M548T substitution to be highly deleterious to protein function, with a SIFT score of 0 . These results suggest that this T-ALL may have developed on a background of DNMT3A-mutated clonal hematopoiesis, and that the other genetic alterations, including the second DNMT3A mutation, were acquired at leukemic transformation.

In order to extend this analysis of non-leukemic DNMT3A mutation, we performed immunophenotypic sorting of two further diagnostic bone marrow samples, and extracted DNA from both the leukemic and the minor residual non-leukemic fractions. We detected a mutation in non-leukemic DNA in one patient (case 4 in Online Supplementary Table S3). Again, we confirmed that this mutation has not been reported as a polymorphism, and that the resultant $\mathrm{P} 385 \mathrm{~L}$ substitution is predicted to damage protein function, with a SIFT score of 0.02. Similar to the case with mutated remission DNA, this sample was negative for two NOTCH1 alterations detected at T-ALL diagnosis, confirming the specificity of DNMT3A mutation persistence (Figure 2B). The other tested nonleukemic DNA had a DNMT3A WT genotype (Online Supplementary Figure S2), giving an overall rate of nonleukemic DNMT3A mutant positivity of $2 / 5$ samples from the GRAALL-2003 and -2005 studies. We also tested a fur- ther three T-ALL cases not included in this cohort, but found no evidence of non-leukemic DNMT3A mutation. This gives an overall incidence of possible clonal hematopoiesis in 2/8 T-ALL samples assessed in our laboratory. It was unfortunately not possible to obtain nonhematopoietic tissue from either of these patients, in order to exclude that these alterations were not constitutional, and to confirm definitively that these results reflect the persistence of a DNMT3A-mutated clonal hematopoietic population in these cases.

\section{DNMT3A mutations are associated with older age and treatment resistance}

A clinico-biological comparison of cases with and without DNMT3A mutations is shown in Table 1. In keeping with previous reports, ${ }^{11,12}$ patients with mutations were considerably older than the rest of the T-ALL cohort (median age 43.9 years mutated vs. 29.4 years non-mutated, $P<0.001)$. In addition, DNMT3A-mutated leukemias were more likely to have an immature T-receptor genotype $^{32}(53.3 \%$ mutated vs. $24.4 \%$ non-mutated, $P=0.016)$, although this did not correspond to a significantly higher incidence of an ETP-ALL immunophenotype ${ }^{33}(35.7 \%$ mutated vs. $20.3 \%$ non-mutated, $P=0.184$ ).

DNMT3A mutation was notably associated with poor initial treatment response. We observed trends towards early corticosteroid resistance $(66.7 \%$ mutated vs. $43.3 \%$ non-mutated, $P=0.081)$ and induction failure $(13.3 \%$ vs. $2.9 \%, P=0.096$, and patients with DNMT3A mutations had significantly higher rates of death during induction (16.7\% vs. $2.8 \%, P=0.027$ ), and lower attainment of complete remission $(72.2 \%$ mutated vs. $94.4 \%$ non-mutated, $P=0.006$ ). As only four patients with mutations were evaluated for minimal residual disease, we could not verify that molecular remission was similarly compromised.

We found that the type of DNMT3A mutation did not significantly correlate with any individual clinico-biological parameter, suggesting that the alterations detected in this study are likely to have broadly similar biological consequences.

\section{DNMT3A mutation correlates with poor outcome in T-cell acute lymphoblastic leukemia}

The median follow-up of the cohort was 5.5 years. Prognostic analyses revealed that DNMT3A mutation was associated with an increased 5-year cumulative incidence of relapse $(53.9 \%$ mutated $v s .28 .7 \%$ non-mutated, $P=0.037$ ) (Figure $3 \mathrm{~A}$ ) and with 5-year event-free survival $[27.8 \%$ mutated vs. $61.0 \%$ non-mutated; hazard ratio (HR) 3.22, (95\% confidence interval (95\% CI): 1.81-5.72, $P<0.001$ ] (Figure 3B). Patients with DNMT3A mutations also had a markedly inferior 5 -year overall survival $(38.8 \%$ mutated vs. $68.7 \%$ non-mutated, HR 2.91, 95\% CI: $1.56-$ 5.43, $P=0.001$ ) (Figure 3C).

\section{The poor prognosis of DNMT3A-mutated T-cell acute lymphoblastic leukemia is age-independent}

Our and others' data ${ }^{11,12}$ have shown that the incidence of DNMT3A mutation in T-ALL increases with age, but previous reports have not documented whether this factor contributes to prognosis. As older patients treated during the GRAALL studies had worse outcomes due to impaired tolerance of intensive chemotherapy, ${ }^{34}$ we considered it critical to determine to what extent age was a confounding prognostic variable. 
We therefore performed bivariate analyses of the effects of DNMT3A mutations and age across a series of outcome measures. These results are shown in Online Supplementary Table S4. In each case, DNMT3A genotype was still associated with significantly increased cumulative incidence of relapse (HR 2.80, 95\% CI: 1.12-6.97, $P=0.034$ ), and shorter event-free survival (HR 2.62, 95\% CI: 1.45-5.06, $P=0.004$ ) and overall survival (HR 2.05, 95\% CI: 1.02-4.12, $P=0.043)$.

Since DNMT3A alterations were almost exclusively found in patients $>40$ years (16/18 cases), we also performed survival analyses that were restricted to the $>40$ year old subgroup, which constituted a quarter of the total cohort of patients $(50 / 198,25.3 \%)$. Consistent with the results of the bivariate analyses, DNMT3A mutation was associated with significantly worse 5-year cumulative incidence of relapse $(58.3 \%$ mutated vs. $21.7 \%$ nonmutated, HR 3.90, 95\% CI: 1.30-11.68, $P=0.015$ ) (Figure 4A), 5-year event-free survival (25.0\% mutated vs. $56.7 \%$ non-mutated, HR 2.95, 95\% CI: 1.37-6.32, $P=0.005)$ (Figure 4B), and 5-year overall survival (37.5\% mutated vs. $62.1 \%$ non-mutated, HR 2.35, 95\% CI: 1.05-5.26, $P=0.038$ ) (Figure 4C).

Finally, we carried out multivariate outcome analyses in the whole cohort using the risk factors that were used to stratify treatment during the GRAALL-2003 and -2005 studies, and which were found to significantly predict prognosis in the univariate analyses. Among age, $\log _{(\mathrm{WBC})}$,
A

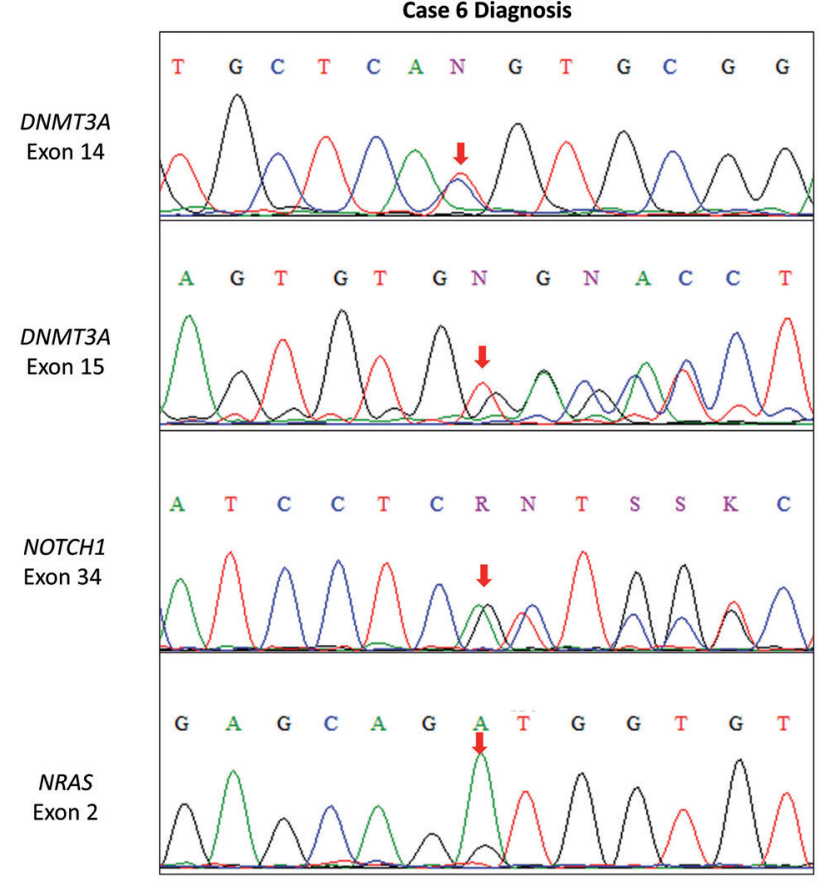

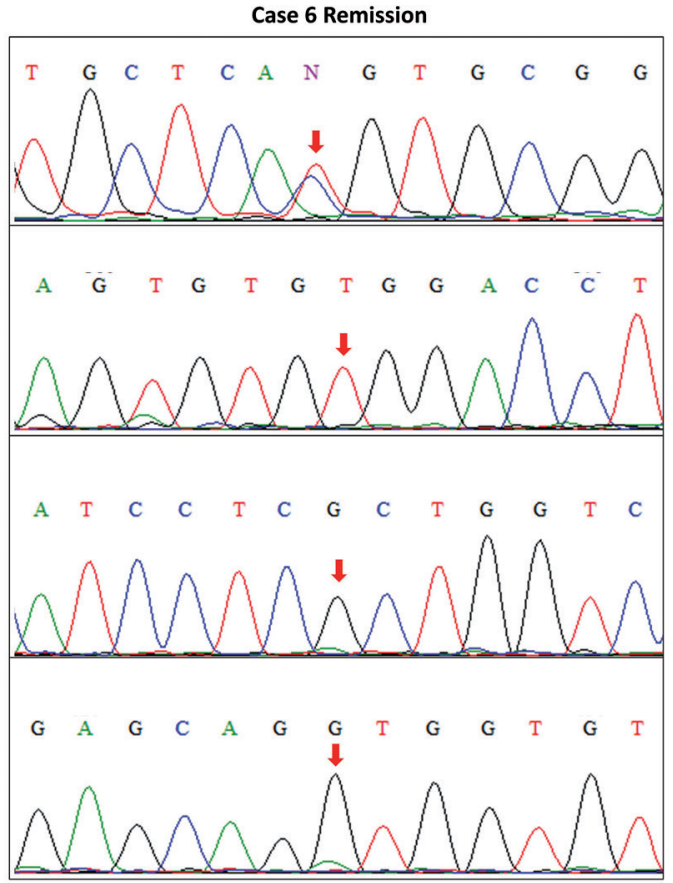

B

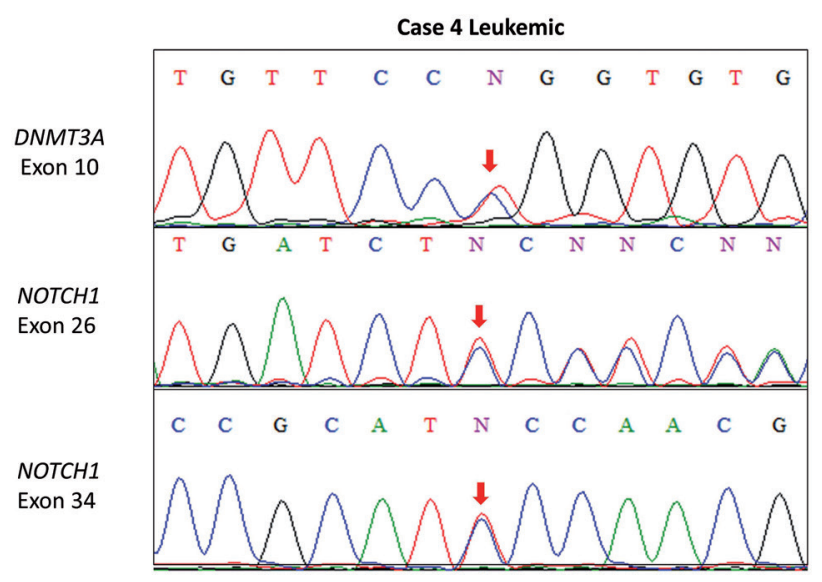

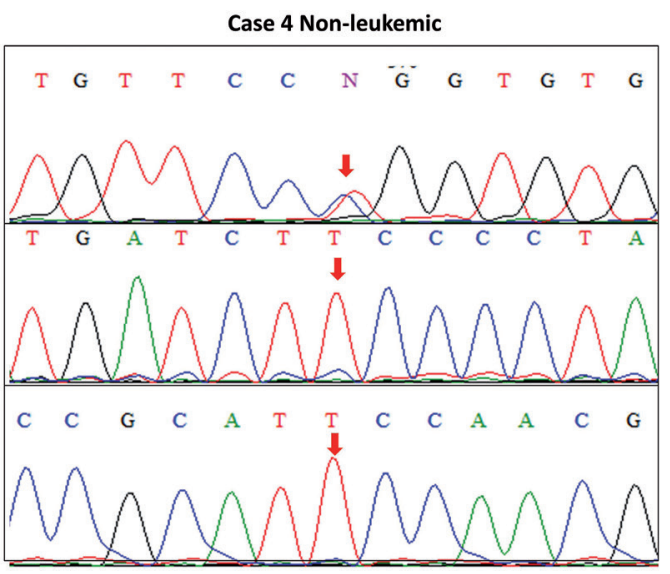

Figure 2. Evidence of DNMT3A mutations in non-leukemic DNA. (A) Direct sequencing of DNMT3A exons 14 and 15 , NOTCH1 and NRAS in diagnostic (left panels) and remission (right panels) samples. (B) Mutational assessment of DNA extracted from leukemic and non-leukemic fractions of samples from patients with T-cell acute lymphoblastic leukemia. Sequencing results of DNMT3A and NOTCH1 in leukemic (left panels) and non-leukemic (right panels) DNA are shown. Cases are numbered according to the listing in Online Supplementary Table S3. 
corticosteroid sensitivity, early chemosensitivity, and DNMT3A genotype, only DNMT3A genotype was associated with cumulative incidence of relapse in univariate analysis (data not shown). As shown in Tables 2 and 3, age, $\log _{(\mathrm{NBC})}$, corticosteroid resistance along with DNMT3A genotype were significantly associated with a poor eventfree survival and overall survival. In multivariate analysis adjusting for these covariates, DNMT3A mutation was still significantly associated with shorter event-free survival (HR 2.33, 95\% CI: 1.06-4.04, $P=0.02$ ) (Table 2), although not with overall survival (HR 1.66, 95\% CI: 0.82-3.37, $P=0.16$ ) (Table 3).

Taken together, these results provide strong evidence that DNMT3A mutation, while mostly observed in older cases, predicts a poor prognosis that is not related to the patient's age.

\section{Discussion}

To our knowledge, this is the most extensive study of DNMT3A-mutated T-ALL yet reported. Our targeted NGS approach allowed comprehensive assessment of genotype across the entire DNMT3A locus, along with the prevalence of co-occurring genetic alterations. Our data additionally benefit from the analysis of a large cohort of patients who were uniformly treated as part of the GRAALL-2003 and 2005 studies, thereby allowing rigorous outcome comparisons between mutated and wild-type cases.

Some of our results were expected, and the findings that
DNMT3A mutations are more commonly present in older patients and genotypically immature leukemias are consistent with previously published data. ${ }^{9,11-13}$ We did not, however, observe increased rates of ETP-ALL immunophenotype, as might have been predicted. We did not detect a clear association with any other geneticallydefined subgroup, and there was no link to increased HOXA expression, which we have previously shown to predict outcome in immature T-ALL. ${ }^{35}$

The detection of DNMT3A alterations in non-leukemic bone marrow suggests that some of these cases of T-ALL might have arisen from DNMT3A-mutated clonal hematopoiesis. While pre-leukemic NOTCH1 mutations have been detected in neonatal blood spot samples of pediatric patients with T-ALL, ${ }^{36}$ to our knowledge our data provide the first potential evidence of age-related clonal hematopoiesis in T-ALL. As it was not possible to obtain non-hematopoietic tissue from either of the patients with this finding, we cannot definitively exclude that these alterations are constitutional, or might even represent an inherited cancer predisposition. Further work is necessary to investigate the incidence of clonal hematopoiesis linked to alterations in DNMT3A and other genes in T-ALL.

Non-leukemic DNMT3A mutations have been seen in $\mathrm{AML},{ }^{30,31}$ and it has been postulated that DNMT3A-altered immature T-ALL might arise from malignant transformation of a multipotent myeloid/lymphoid progenitor cell. ${ }^{12}$ In keeping with this, one of the cases with a non-leukemic DNMT3A mutation in this study had expression of myeloid cell surface markers as part of an ETP-ALL phe-

Table 1. Characteristics and outcome of the patients according to DNMT3A genotype.

\begin{tabular}{|c|c|c|c|c|}
\hline & DNMT3A Mutated & DNMT3A WHid-type & Total & P-value \\
\hline Total (\%) & $18(9.1 \%)$ & $180(90.9 \%)$ & $198(100 \%)$ & \\
\hline $\begin{array}{l}\text { Clinical subsets analyzed } \\
\text { Male } \\
\text { Median age (years) }[\mathrm{IQR}] \\
\text { WBC }\left(10^{\circ} / \mathrm{L} \text {, median) }\right. \\
\text { CNS involvement }\end{array}$ & $\begin{array}{c}13(72.2 \%) \\
43.9[40.7-53.6] \\
41.1 \\
3(16.7 \%)\end{array}$ & $\begin{array}{c}128(71.1 \%) \\
29.4[23.2-37.2] \\
31.9 \\
21(11.7 \%)\end{array}$ & $\begin{array}{c}141(71.2 \%) \\
30.5[23.4-40.4] \\
32.6 \\
24(12.1 \%)\end{array}$ & $\begin{array}{c}0.921 \\
<\mathbf{0 . 0 0 1} \\
0.491 \\
0.463\end{array}$ \\
\hline $\begin{array}{l}\text { T-cell receptor status } \\
\text { Immature (IM0, IMD, IMG') } \\
\alpha \beta \text { lineage } \\
\gamma \delta \text { lineage }\end{array}$ & $\begin{array}{l}8(53.3 \%) \\
3(20.0 \%) \\
4(26.7 \%)\end{array}$ & $\begin{array}{c}38(24.4 \%) \\
104(66.7 \%) \\
14(9.0 \%)\end{array}$ & $\begin{array}{l}46(26.9 \%) \\
107(62.6 \%) \\
18(10.5 \%)\end{array}$ & $\begin{array}{c}0.015 \\
<0.001 \\
0.033\end{array}$ \\
\hline ETP immunophenotype ${ }^{\#}$ & $5(35.7 \%)$ & $32(20.3 \%)$ & $37(18.7 \%)$ & 0.184 \\
\hline $\begin{array}{l}\text { Oncogenetics } \\
\text { HOXA positivity } \\
\text { NOTCH1/FBXW7 mutated } \\
\text { RAS/PTEN mutated } \\
\text { Risk classifier, high }\end{array}$ & $\begin{array}{l}4(25.0 \%) \\
15(83.3 \%) \\
5(29.4 \%) \\
8(44.4 \%)\end{array}$ & $\begin{array}{l}41(26.6 \%) \\
124(68.9 \%) \\
33(19.4 \%) \\
74(42.3 \%)\end{array}$ & $\begin{array}{l}45(26.5 \%) \\
139(70.2 \%) \\
38(20.3 \%) \\
82(42.5 \%)\end{array}$ & $\begin{array}{l}1.000 \\
0.282 \\
0.365 \\
1.000\end{array}$ \\
\hline $\begin{array}{l}\text { Early treatment response } \\
\text { Corticosteroid sensitivity } \\
\text { Complete remission } \\
\text { Induction death } \\
\text { Induction failure }\end{array}$ & $\begin{array}{c}6(33.3 \%) \\
13(72.2 \%) \\
3(16.7 \%) \\
2 / 15(13.3 \%)\end{array}$ & $\begin{array}{c}102(56.7 \%) \\
170(94.4 \%) \\
5(2.8 \%) \\
5 / 175(2.9 \%)\end{array}$ & $\begin{array}{c}108(54.5 \%) \\
183(92.4 \%) \\
8(4.0 \%) \\
7 / 190(3.7 \%)\end{array}$ & $\begin{array}{c}0.081 \\
\mathbf{0 . 0 0 6} \\
\mathbf{0 . 0 2 7} \\
0.097\end{array}$ \\
\hline $\begin{array}{l}\text { 5-year treatment outcome } \\
\text { Cumulative incidence of relapse } \\
\text { Event-free survival } \\
\text { Overall survival }\end{array}$ & $\begin{array}{l}53.9 \% \\
27.8 \% \\
38.8 \%\end{array}$ & $\begin{array}{l}28.7 \% \\
61.0 \% \\
68.7 \%\end{array}$ & $\begin{array}{c}30.5 \% \\
58 \% \\
66 \%\end{array}$ & $\begin{array}{c}0.037 \\
<0.001 \\
0.001\end{array}$ \\
\hline
\end{tabular}

"T-cell receptor status (n=171), early thymic precursor (ETP) immunophenotype (n=172), HOXA positivity (n=170) and Risk classifier based on NOTCH1, FBXW7, PTEN, NRAS and KRAS genotypes $(\mathrm{n}=193)$ were determined as previously described ${ }^{32,33,35,37}$ For the Risk classifier, numbers categorized as high risk (NOTCH1/FBXW7 WT and/or NRAS/KRAS/PTEN altered) are shown. Statistically significant results are shown in bold. 
A

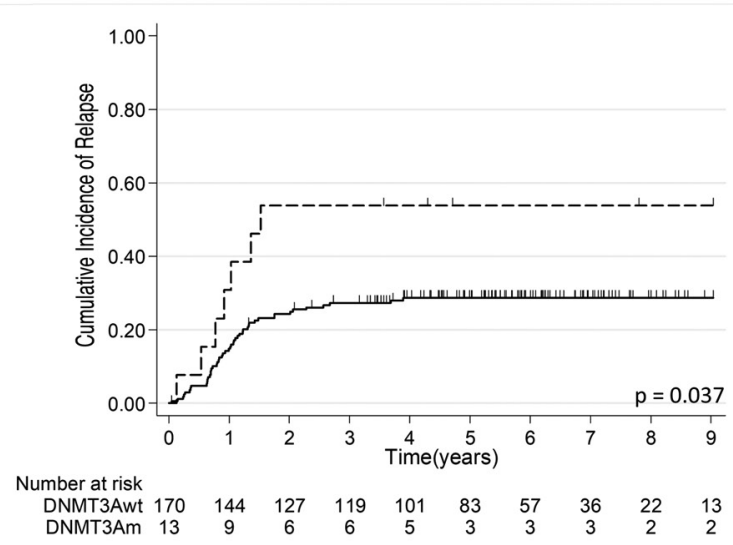

C

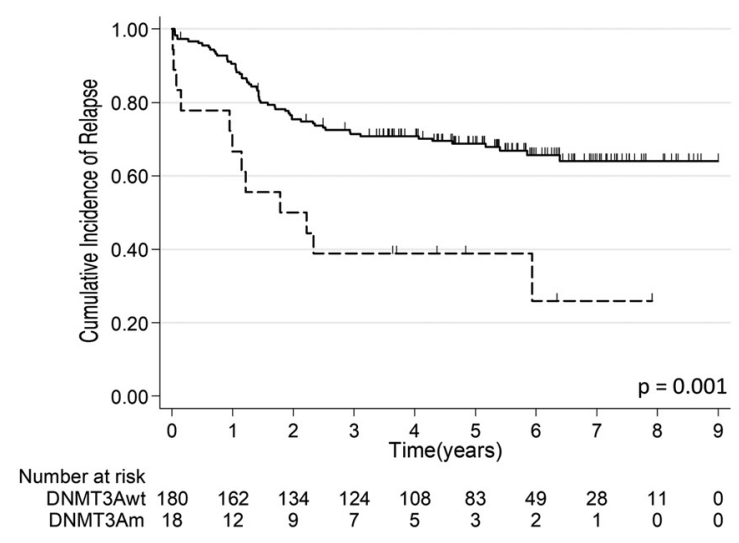

A

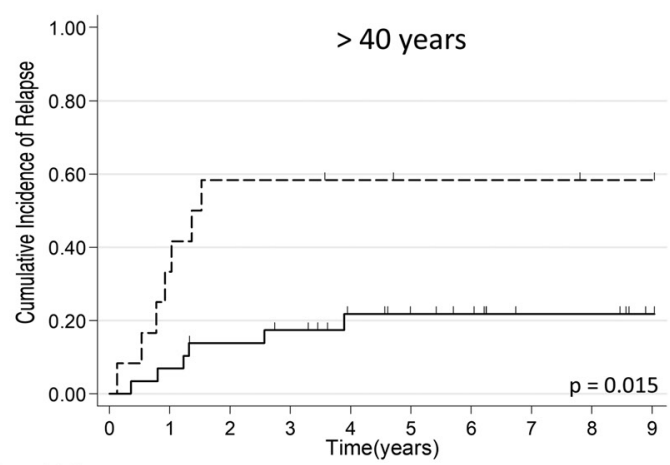

Number at risk

$\begin{array}{lcccccccccc}\text { DNMT3Awt } & 29 & 27 & 24 & 22 & 17 & 14 & 12 & 7 & 7 & 3 \\ \text { DNMT3Am } & 12 & 8 & 5 & 5 & 4 & 3 & 3 & 3 & 2 & 2\end{array}$

C

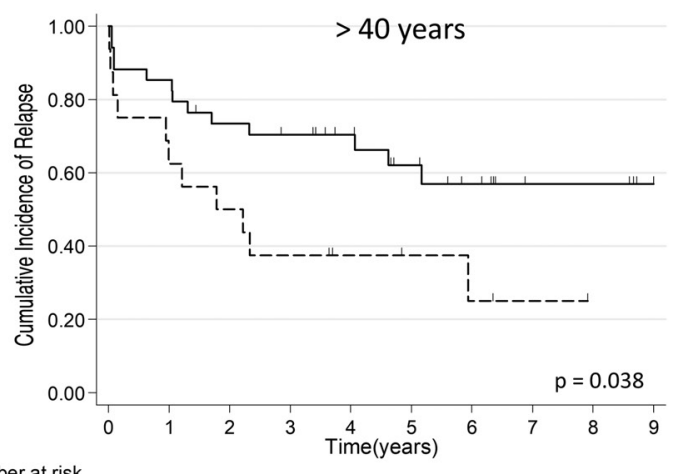

Number at risk

$\begin{array}{lcccccccccc}\text { DNMT3Awt } & 34 & 29 & 24 & 22 & 18 & 13 & 9 & 4 & 4 & 0 \\ \text { DNMT3Am } & 16 & 10 & 8 & 6 & 4 & 3 & 2 & 1 & 0 & 0\end{array}$
B

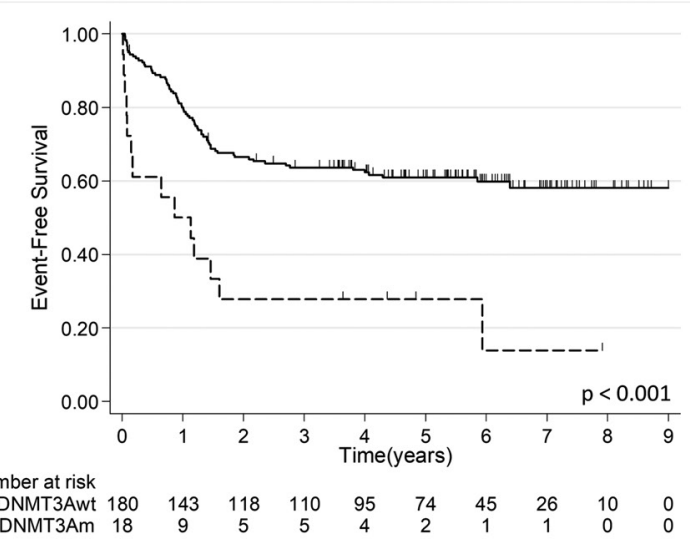

Figure 3. DNMT3A mutation correlates with poor outcome in T-cell acute lymphoblastic leukemia. Comparisons of outcomes for patients with $(n=18)$ and without $(n=180)$ DNMT3A mutations are shown for: $(A)$ cumulative incidence of relapse; (B) event-free survival; and $(C)$ overall survival. The 5-year results were as follows: cumulative incidence of relapse $53.9 \%$ mutated vs. $28.7 \%$ nonmutated; event-free survival $27.8 \%$ mutated vs. $61.0 \%$ non-mutated; overall survival $38.8 \%$ mutated vs. $68.7 \%$ non-mutated. $P$ values are indicated.

B

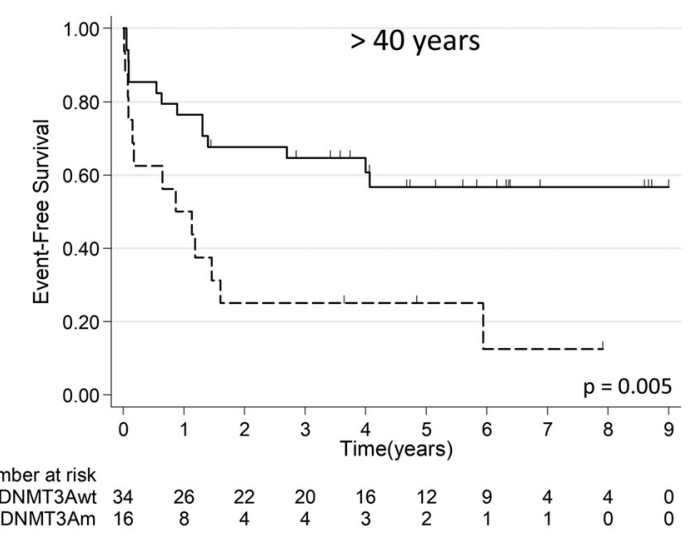

Figure 4. DNMT3A genotype predicts outcome in the age group of patients at risk of mutation. Comparisons of outcomes for patients with $(n=16)$ and without $(n=34)$ mutations in patients $>40$ years are shown for: (A) cumulative incidence of relapse; (B) event-free survival; and $(C)$ overall survival. The 5 -year results were as follows: cumulative incidence of relapse $58.3 \%$ mutated vs. $21.7 \%$ nonmutated; event-free survival, $25.0 \%$ mutated vs. $56.7 \%$ non-mutated; overall survival $37.5 \%$ mutated vs. $62.1 \%$ non-mutated. $P$ values are indicated. 
Table 2. Prognostic impact of DNMT3A genotype on event-free survival.

\begin{tabular}{|c|c|c|c|c|c|c|}
\hline \multirow[t]{2}{*}{ EFS } & \multicolumn{3}{|c|}{ Univariate } & \multicolumn{3}{|c|}{ Multivariate } \\
\hline & HR & $95 \%$ Cl & $\boldsymbol{P}$ & HR & $95 \%$ CI & $\boldsymbol{P}$ \\
\hline Age* & 1.03 & $1.01-1.05$ & 0.009 & 1.02 & $1.00-1.04$ & 0.071 \\
\hline $\log _{(\mathrm{WBC})} *$ & 1.62 & $1.12-2.34$ & 0.011 & 1.50 & $0.98-2.29$ & 0.062 \\
\hline Corticosteroid sensitivity & 0.52 & $0.34-0.81$ & 0.003 & 0.66 & $0.41-1.07$ & 0.093 \\
\hline Early chemosensitivity & 0.90 & $0.68-1.18$ & 0.436 & - & - & - \\
\hline DNMT3A mutation & 3.22 & $1.81-5.72$ & $<0.001$ & 2.20 & $1.13-4.27$ & 0.02 \\
\hline
\end{tabular}

*Continuous variable. Statistically significant differences are highlighted in bold. EFS: event-free survival; HR: hazard ratio; $95 \%$ CI: $95 \%$ confidence interval; WBC: white blood cell count..

Table 3. Prognostic impact of DNMT3A genotype on overall survival.

\begin{tabular}{|c|c|c|c|c|c|c|}
\hline \multirow[t]{2}{*}{ OS } & \multicolumn{3}{|c|}{ Univariate } & \multicolumn{3}{|c|}{ Multivariate } \\
\hline & $H R$ & $95 \%$ CI & p & HR & $95 \%$ CI & p \\
\hline Age* $^{*}$ & 1.04 & $1.01-1.06$ & 0.002 & 1.03 & $1.01-1.06$ & 0.009 \\
\hline $\log _{(\mathrm{NBCC}} *$ & 1.65 & $1.10-2.46$ & 0.015 & 1.63 & $1.03-2.57$ & 0.037 \\
\hline Corticosteroid sensitivity & 0.59 & $0.37-0.94$ & 0.027 & 0.79 & $0.47-1.34$ & 0.388 \\
\hline Early chemosensitivity & 0.94 & $0.71-1.24$ & 0.640 & - & - & - \\
\hline DNMT3A mutation & 2.91 & $1.56-5.43$ & 0.001 & 1.66 & $0.82-3.37$ & 0.160 \\
\hline
\end{tabular}

${ }^{*}$ Continuous variable. Statistically significant differences are highlighted in bold. OS: overall survival; HR: hazard ratio; 95\% CI: 95\% confidence interval; WBC: white blood cell count..

notype, while full immunophenotypic assessment was unfortunately not possible for the other patient. The factors that may dictate the acute leukemic phenotype in clonally mutated cases remain to be clarified. For example, this might be influenced by the differentiation capacity of the cell in which the initial DNMT3A mutation occurs. In addition, it is tempting to speculate that the acquisition of specific cooperative mutations, such as the NOTCH1 mutations observed in these T-ALL cases, might act as lineage determinants.

Outcome analyses revealed that DNMT3A mutation correlated with poor prognosis independently of the patients' age in bivariate analyses. Multivariable analyses using parameters that were used to stratify treatment in the GRAALL-2003 and -2005 studies showed that DNMT3A genotype independently predicted both event-free survival and cumulative incidence of relapse. DNMT3A mutation status also independently predicted event-free survival and overall survival in bivariable analyses that incorporated our recently described oncogenetic risk classifier ${ }^{37}$ (Online Supplementary Table S5). These results suggest that DNMT3A mutation is directly linked to aggressive T-ALL biology. As DNMT3A-altered T-ALL had higher mutation rates in other genes included in our targeted sequencing panel, it is also possible that increased genotype complexity may contribute to the more aggressive phenotype in these leukemias. This issue may be clarified by more comprehensive genomic assessment in future studies.

The high rates of treatment failure observed in this study suggest that therapeutic intervention is warranted for DNMT3A-mutated cases, and that treatment intensification should be considered for the infrequent younger patients with mutations. Indeed, we have previously documented a benefit from allogeneic stem cell transplantation in first complete remission for ETP-ALL, ${ }^{24}$ which similarly exhibits high rates of intrinsically treatment-resistant disease. As only three of the 18 DNMT3A-mutated patients in this study underwent allogeneic stem cell transplantation (data not shown), we are unable to estimate the potential benefit of such treatment in this setting. We recently reported that treatment-related toxicity in the GRAALL-2005 study increased in proportion to the patients' age ${ }^{38}$ and further therapy intensification in elderly patients must therefore be considered of questionable benefit. The upper age of this study cohort was 60 years, but it is likely that the rate of mutations in older patients who do not tolerate such intensive chemotherapy is higher. Data reported for patients with AML suggest that DNMT3A mutation confers increased sensitivity to hypomethylating agents, ${ }^{39}$ providing a rationale for evaluation of these drugs in DNMT3A-mutated T-ALL. In the longer term, it is to be hoped that investigation of the molecular mechanisms by which DNMT3A mutation alters T-ALL biology will lead to better treatments and improved outcomes for these high-risk cases.

\section{Acknowledgments}

This manuscript was written on behalf of the Group for Research on Adult Acute Lymphoblastic Leukemia (GRAALL), which includes the former France-Belgium Group for Lymphoblastic Acute Leukemia in Adults (LALA), the French Western-Eastern Group for Lymphoblastic Acute Leukemia (GOELAL), and the Swiss Group for Clinical Cancer Research (SAKK). The authors would like to thank all participants in the GRAALL-2003 and GRAALL-2005 study groups for collection and provision of data and samples, and $V$. Lheritier for collection of clinical data. The GRAALL-2003 study was sponsored by the Hoppitaux de Toulouse, and the GRAALL-2005 study by the Assistance Publique-Hôpitaux de Paris. The SAKK was supported by the Swiss State Secretariat for Education, Research and Innovation (SERI). JB was supported by a Kay Kendall Leukaemia Fund Intermediate Research Fellowship and by the National Children's Research Centre, Children's Health Ireland at Crumlin, Dublin, Ireland. The Necker Laboratory is supported by the Association Laurette Fugain, La Ligue contre le Cancer and the INCa CARAMELE Translational Research and PhD programs. 


\section{References}

1. Ley TJ, Ding L, Walter MJ, et al. DNMT3A mutations in acute myeloid leukemia. N Engl J Med. 2010;363(25):2424-2433.

2. Renneville A, Boissel N, Nibourel O, et al. Prognostic significance of DNA methyltransferase $3 \mathrm{~A}$ mutations in cytogenetically normal acute myeloid leukemia: a study by the Acute Leukemia French Association. Leukemia. 2012;26(6):1247-1254.

3. Gale RE, Lamb K, Allen C, et al. Simpson's paradox and the impact of different DNMT3A mutations on outcome in younger adults with acute myeloid leukemia. J Clin Oncol. 2015;33(18):20722083.

4. Marcucci G, Metzeler KH, Schwind S, et al. Age-related prognostic impact of different types of DNMT3A mutations in adults with primary cytogenetically normal acute myeloid leukemia. J Clin Oncol. 2012;30(7): 742-750.

5. Yan XJ, Xu J, Gu ZH, et al. Exome sequencing identifies somatic mutations of DNA methyltransferase gene DNMT3A in acute monocytic leukemia. Nat Genet. 2013;43(4):309-315.

6. Haferlach T, Nagata Y, Grossmann V, et al. Landscape of genetic lesions in 944 patients with myelodysplastic syndromes. Leukemia. 2014;28(2):241-247.

7. Stegelmann F, Bullinger L, Schlenk RF, et al. DNMT3A mutations in myeloproliferative neoplasms. Leukemia. 2011;25(7):12171219

8. Jankowska AM, Makishima H, Tiu RV, et al. Mutational spectrum analysis of chronic myelomonocytic leukemia includes genes associated with epigenetic regulation: UTX, EZH2, and DNMT3A. Blood. 2011;118(14): 3932-3941

9. Roller A, Grossmann V, Bacher U, et al. Landmark analysis of DNMT3A mutations in hematological malignancies. Leukemia. 2013;27(7):1573-1578.

10. Van Vlierberghe P, Ambesi-Impiombato A, Perez-Garcia A, et al. ETV6 mutations in early immature human $T$ cell leukemias. J Exp Med. 2011;208(13):2571-2579

11. Grossmann V, Haferlach C, Weissmann S, et al. The molecular profile of adult T-cell acute lymphoblastic leukemia: mutations in RUNX1 and DNMT3A are associated with poor prognosis in T-ALL. Genes Chromosomes Cancer. 2013;52(4):410-422.

12. Neumann M, Heesch S, Schlee C et al. Whole-exome sequencing in adult ETP-ALL reveals a high rate of DNMT3A mutations. Blood. 2013;121(23):4749-4752.

13. Van Vlierberghe P, Ambesi-Impiombato A, De Keersmaecker K, et al. Prognostic relevance of integrated genetic profiling in adult T-cell acute lymphoblastic leukemia. Blood.
2013;122(1):74-82.

14. Couronne L, Bastard C, Bernard OA. TET2 and DNMT3A mutations in human T-cell lymphoma. N Engl J Med. 2012;366(1):9596.

15. Sakata-Yanagimoto M, Enami T, Yoshida K, et al. Somatic RHOA mutation in angioimmunoblastic T cell lymphoma. Nat Genet. 2014;46(2):171-175.

16. Choi J, Goh G, Walradt T, et al. Genomic landscape of cutaneous $\mathrm{T}$ cell lymphoma. Nat Genet. 2015;47(9):1011-1019.

17. Ho PA, Kutny MA, Alonzo TA, et al. Leukemic mutations in the methylationassociated genes DNMT3A and IDH2 are rare events in pediatric AML: a report from the Children's Oncology Group. Pediat Blood Cancer. 2011;57(2):204-209.

18. Huether R, Dong L, Chen X, et al. The landscape of somatic mutations in epigenetic regulators across 1,000 paediatric cancer genomes. Nat Commun. 2014:5:3630.

19. Shiba N, Taki T, Park MJ, et al. DNMT3A mutations are rare in childhood acute myeloid leukaemia, myelodysplastic syndromes and juvenile myelomonocytic leukaemia. Br J Haematol. 2012;156(3):413414.

20. Shen Y, Zhu YM, Fan X, et al. Gene mutation patterns and their prognostic impact in a cohort of 1185 patients with acute myeloid leukemia. Blood. 2011;118(20): 5593-5603

21. Ribeiro AF, Pratcorona M, Erpelinck Verschueren C, et al. Mutant DNMT3A: a marker of poor prognosis in acute myeloid leukemia. Blood 2012;119(24):5824-5831.

22. Tie R, Zhang $\mathrm{T}$, Fu $\mathrm{H}$, et al. Association between DNMT3A mutations and prognosis of adults with de novo acute myeloid leukemia: a systematic review and metaanalysis. PloS One. 2014;9(6):e93353.

23. Zhang J, Ding L, Holmfeldt L, et al. The genetic basis of early $\mathrm{T}$-cell precursor acute lymphoblastic leukaemia. Nature. 2012:481(7380):157-163.

24. Bond J, Graux C, Lhermitte L, et al. Early response-based therapy stratification improves survival in adult early thymic precursor acute lymphoblastic leukemia: group for research on adult acute lymphoblastic leukemia study. J Clin Oncol. 2017;35(23):2683-2691

25. Papaemmanuil E, Gerstung M, Bullinger L, et al. Genomic classification and prognosis in acute myeloid leukemia. N Engl J Med. 2016;374(23):2209-2221.

26. Lin ME, Hou HA, Tsai CH, et al. Dynamics of DNMT3A mutation and prognostic relevance in patients with primary myelodysplastic syndrome. Clin Epigenetics. 2018;10:42.

27. Genovese G, Kahler AK, Handsaker RE, et al. Clonal hematopoiesis and blood-cancer risk inferred from blood DNA sequence. $\mathrm{N}$
Engl J Med. 2014;371(26):2477-2487.

28. Jaiswal S, Fontanillas P, Flannick J, et al. Agerelated clonal hematopoiesis associated with adverse outcomes. N Engl J Med. 2014;371(26):2488-2498.

29. Xie M, Lu C, Wang J, et al. Age-related mutations associated with clonal hematopoietic expansion and malignancies. Nat Med. 2014;20(12):1472-1478

30. Shlush LI, Zandi S, Mitchell A, et al. Identification of pre-leukaemic haematopoietic stem cells in acute leukaemia. Nature. 2014;506(7488):328-333.

31. Corces-Zimmerman MR, Hong WJ, Weissman IL, Medeiros BC, Majeti R. Preleukemic mutations in human acute myeloid leukemia affect epigenetic regulators and persist in remission. Proc Natl Acad Sci U S A. 2014;111(7):2548-2553.

32. Asnafi V, Beldjord K, Boulanger E, et al. Analysis of TCR, pT alpha, and RAG-1 in T acute lymphoblastic leukemias improves understanding of early human T-lymphoid lineage commitment. Blood. 2003;101(7): 2693-2703.

33. Coustan-Smith E, Mullighan CG, Onciu M et al. Early T-cell precursor leukaemia: a subtype of very high-risk acute lymphoblastic leukaemia. Lancet Oncol. 2009;10(2):147 156

34. Huguet F, Leguay T, Raffoux E, et al. Pediatric-inspired therapy in adults with Philadelphia chromosome-negative acute lymphoblastic leukemia: the GRAALL-2003 study. J Clin Oncol. 2009;27(6):911-918.

35. Bond J, Marchand T, Touzart A, et al. An early thymic precursor phenotype predicts outcome exclusively in HOXA-overexpressing adult $\mathrm{T}$-cell acute lymphoblastic leukemia: a Group for Research in Adult Acute Lymphoblastic Leukemia study. Haematologica. 2016;101(6):732-740.

36. Eguchi-Ishimae M, Eguchi M, Kempski $\mathrm{H}$, Greaves M. NOTCH1 mutation can be an early, prenatal genetic event in T-ALL Blood. 2008;111(1):376-378.

37. Trinquand A, Tanguy-Schmidt A, Ben Abdelali R, et al. Toward a NOTCH1/FBXW7/RAS/PTEN-based oncogenetic risk classification of adult $\mathrm{T}$-cell acute lymphoblastic leukemia: a Group for Research in Adult Acute Lymphoblastic Leukemia study. J Clin Oncol. 2013:31 (34):4333-4342

38. Huguet Fo, Leguay $T$, Thomas $X$, et al. The upper age limit for a pediatric-inspired therapy in younger adults with $\mathrm{Ph}$-negative acute lymphoblastic leukemia (ALL)? Analysis of the GRAALL-2005 study. 2016:762-762

39. Metzeler KH, Walker A, Geyer S, et al DNMT3A mutations and response to the hypomethylating agent decitabine in acute myeloid leukemia. Leukemia. 2012;26(5): 1106-1107. 